\title{
AVANCE AL ESTUDIO DE LAS CERÁMICAS PERTENECIENTES AL NEOLÍTICO ANTIGUO AVANZADO DE LOS CASTILLEJOS (MONTEFRÍO, GRANADA)*
}

\author{
Progress in the study of the pottery belonging to the Ancient Advanced Neolitbic \\ of Los Castillejos (Montefrio, Granada)
}

\author{
María Teresa BlázQuez GonzÁLez \\ Universidad de Granada \\ E-mail: maite.mbgop@hotmail.com
}

Fecha de recepción: 6-III-2011

Fecha de aceptación: 31-III-2011

\begin{abstract}
RESUMEN: El contenido de este artículo es un avance del estudio que se está realizando de las cerámicas decoradas pertenecientes a las cuatro primeras fases del periodo I de la estratigrafía del yacimiento conocido como Los Castillejos (Montefrío, Granada), es decir, de la fase que ha sido datada dentro del Neolítico Antiguo Avanzado (5400-5150 cal a.C.). Dicho estudio se inició motivado por el interés de analizar las piezas cerámicas de esta etapa que habían sido localizadas en las excavaciones realizadas entre 1991 y 1994, y que habían permanecido inéditas hasta la fecha. Con este fin y para estudiar la tecnología cerámica se ha utilizado una metodología que abarca el estudio historiográfico del yacimiento, la localización, cuantificación y pesado de las piezas, el escaneado y dibujo de las cerámicas, el estudio de las decoraciones y otras características de las arcillas y piezas, así como analíticas de laboratorio cuyos resultados serán publicados en un futuro.
\end{abstract}

Palabras Clave: Neolítico Antiguo Avanzado, estudio cerámico, análisis decorativo, tecnología cerámica, cerámica neolítica.

ABSTRACT: The content of this article is an advance of the study that is being realized about the decorated ceramics belonging to the first four phases of the period I of the stratigraphy of the site known as Los Castillejos (Montefrio, Granada), ie, of the phase that has been dated in the Ancient Advanced Neolithic (5400-5150 cal BC). This study began motivated by the interest in analyzing the ceramics belonging to this stage that were excavated between 1991 and 1994, and that had remained unpublished until recently. To this end and to study the ceramic technology it has been used a methodology that includes the historiographical study of the site, the location, quantification and the weight of the pieces, the scanning and design of the ceramics, the study of the decorations and other characteristics of the ceramics and of the pieces, and laboratory analytical which results will be published in the future.

Keywords: Ancient Advanced Neolithic, ceramic study, decorative analysis, ceramic technology, Neolithic pottery. 


\section{INTRODUCCIÓN}

La cerámica es uno de los materiales arqueológicos que aparece más frecuentemente en los trabajos de excavación. No obstante los estudios que se han hecho de estos restos y la información que de ellos se ha obtenido, varía mucho según la etapa y la corriente historiográfica de la que hablemos. Así en la Edad Media cuando diferentes vasijas afloraban a la superficie durante el transcurso de las labores agrícolas, se pensaba que su origen era de tipo mítico y mágico, y solo unos pocos defendían su manufactura humana ${ }^{1}$.

Durante el siglo XVI se produce un cambio radical con la tendencia anterior. Es el momento del renacer clásico y del avance en el conocimiento del mundo griego y romano, incluyendo en él sus cerámicas, que ya serán introducidas en las primeras colecciones emergentes del momento, las cuales dejan entrever la tendencia que en el siglo XVIII alcanzará su mayor auge, el coleccionismo. Es en esta nueva etapa cuando las piezas cerámicas etruscas, griegas, romanas, etc. acumuladas durante largo tiempo por coleccionistas y anticuarios ven la luz y se realizan publicaciones sobre ellas.

En el siglo XIX aumenta el número de excavaciones y por lo tanto lo mismo sucede con el volumen de material cerámico. Este hecho tiene importantes repercusiones para el desarrollo dela ceramología ya que se potencia el estudio de la tipología, la estratigrafía y la seriación, que influenciadas por las ideas de progreso cultural y evolucionismo se centran en establecer cronologías de valor relativo estudiando los restos arqueológicos, entre ellos las cerámicas, según su morfología, su posición horizontal y vertical dentro del corte de la excavación, y la supuesta evolución gradual de las vasijas pertenecientes a una misma tradición cultural ${ }^{2}$.

Finalmente desde mediados del siglo XX hasta la actualidad los estudios cerámicos, y en general el estudio de todo tipo de material arqueológico, ha sufrido otro cambio importante a destacar. En este caso bajo la influencia de la Nueva Arqueología y de la Arqueología Posprocesual, la descripción y clasificación de vasijas y fragmentos se complementa con los estudios etnográficos y simbólicos, pero sobre todo con nuevas y numerosas técnicas científicas que en general se tienden a agrupar bajo la denominación genérica de Arqueometría ${ }^{3}$.

\footnotetext{
* El presente artículo es un avance al trabajo final del Máster en Arqueología que se imparte en la Universidad de Granada. Dicho trabajo cuenta con Fernando Molina González, Juan Antonio Cámara Serrano y Josefa Capel Martínez como tutores y se incluye dentro del proyecto HAR2008-04577 “Cronología de la consolidación del sedentarismo y la desigualdad social en el Alto Guadalquivir" financiado por el Ministerio de Ciencia e Innovación. 1 ORTON, C., TYERS, P., VINCE, A. (1997), La cerámica en Arqueología, Crítica, Barcelona. pp. 19-20.

2 ORTON, C., TYERS, P., VINCE, A. (1997), op. cit. pp. 20-25; FERNÁNDEZ MARTÍNEZ, V. M. (1989), Teoría y método de la arqueología, Síntesis, Madrid. pp. 22-25. 3 ORTON, C., TYERS, P., VINCE, A. (1997), op. cit. pp. 27.
} 
Estas nuevas técnicas científicas han alcanzado una gran importancia en el desarrollo de los estudios arqueológicos actuales, hasta tal punto que hoy en día parece casi inconcebible visualizar un estudio de materiales que no incluya alguna de ellas. En el caso concreto de los estudios cerámicos análisis como lámina delgada, difracción de rayos $\mathrm{X}$, fluorescencia de rayos $\mathrm{X}$, termoluminiscencia u otras técnicas, ayudan a conocer información sobre temas que antes de su desarrollo parecían imposibles de abarcar, como los componentes minerales de las cerámicas, su origen geológico, las técnicas de manufacturación, su comercio y distribución, la fecha en que se cocieron, conocer el contenido original que tuvieron, o averiguar su posible función según las propiedades físicas de las pastas.

\section{LOCALIZACIÓN}

El poblado prehistórico de Los Castillejos es en verdad un yacimiento con una amplia cronología asentada, sobre todo para las fases prehistóricas, aunque sus restos llegan hasta etapas medievales pasando por el periodo visigodo, la época romana o el mundo ibérico. Dicho yacimiento se localiza aproximadamente a unos $4 \mathrm{Km}$. del término municipal de Montefrío (Granada), donde se ubica concretamente en una elevación geográfica conocida como Las Peñas de Los Gitanos que forman parte de la región de Los Montes, ésta última incluida dentro del conjunto de las sierras subbéticas. ${ }^{4}$.

El relieve de Las Peñas de Los Gitanos destaca por la presencia de los llamados maciños, afloramientos rocosos fácilmente erosionables que han dado lugar a un paisaje de aterrazamientos naturales. Dentro de este conjunto, el poblado prehistórico se localiza en la terraza superior que cuenta con 125 m. de largo por $30 \mathrm{~m}$. de ancho y se eleva a 1050 m.s.n.m ${ }^{5}$. Sin embargo, Los Castillejos no es el único yacimiento localizado dentro de las peñas, sino que por el contrario dentro de ellas hay todo un conjunto arqueológico, nombrado Bien de Interés Cultural en 1996, que incluye varias cuevas que han aportado material prehistórico como Cueva Negra, Cueva de las Cabras, Cueva de las Tontas, Cueva de la Alondra o Cueva Alta ${ }^{6}$. También se incluye

4 ARRIBAS PALAU, A., MOLINA GONZÁLEZ, F. (1977), “El poblado de Los Castillejos en Las Peñas de los Gitanos (Montefrío, Granada). Campañas de excavaciones de 1971 y 1974", XIV Congreso Nacional de Arqueología (Vitoria, 1975), Zaragoza. pp. 389.

5 ARRIBAS PALAU, A., MOLINA GONZÁLEZ, F. (1977), op. cit. pp. 389-390.

6 MERGELINA, C. (1945-1946), "La estación arqueológica de Montefrío (Granada) II. La acrópoli de Guirrete (Los Castillejos)", Boletín del Seminario de Estudios de Arte y Arqueología XII, Valladolid. pp. 15-26.; TARRADELL, M. (1952), "La Edad del Bronce en Montefrío (Granada). Resultados de las excavaciones en yacimientos de las Peñas de los Gitanos", Ampurias XIV, Barcelona. pp. 49-80.; MORENO, Mª A. (1982), "Los materiales arqueológicos del poblado de Los Castillejos y Cueva Alta (Montefrío) procedente de las excavaciones de 1946 y 1947”, Cuadernos de Prehistoria de la Universidad de Granada 7, Granada. pp. 235-266.; TORRE SANTANA, Ma del P. (1984), "La Cueva de las Tontas en la estación arqueológica de Las Peñas de los Gitanos (Montefrío, Granada)", Cuadernos de Prehistoria de la Universidad de Granada 9, Granada. pp. 85-96. 
dentro de Las Peñas de los Gitanos una necrópolis megalítica, que conocida desde mediados del siglo XIX ha sido divida en varias zonas entre las que destacan El Rodeo con sepulcros más grandes y antiguos, y La Camarilla con tumbas de menores dimensiones y más recientes?

\section{CONJUNTO ARQUEOLÓGICO DE LAS PEN̉AS DE LOS GITANOS}

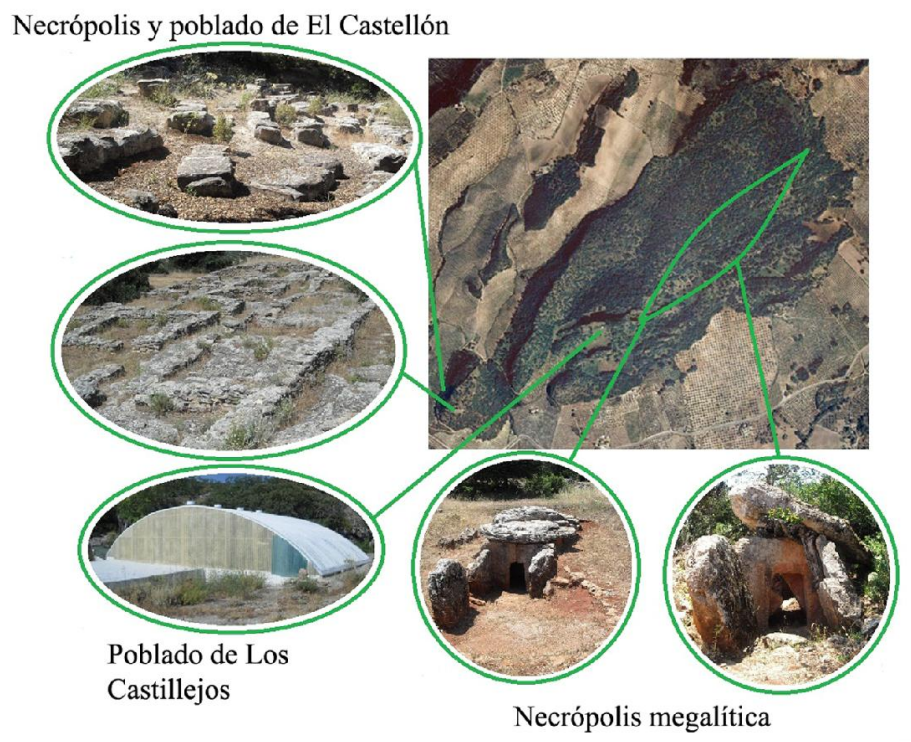

Fig. I Foto aérea de Las Peñas de los Gitanos (imagen obtenida de Google Earth) donde se localizan sus principales yacimientos.

7 GÓNGORA Y MARTÍNEZ, M. de. (1868), Antigüedades prehistóricas de Andalucía, monumentos, inscripciones, armas, utensilios y otros importantes objetos pertenecientes a los tiempos más remotos de su población, Imprenta a cargo de C. Moro, Madrid. pp. 57-88; MERGELINA, C. (1941-1942), "La estación arqueológica de Montefrío (Granada) I. Los dólmenes", Boletín del Seminario de Estudios de Arte y Arqueología VIII (B. S. A. A.), Valladolid. pp. 33-106.; GÓMEZMORENO, M. (1949), "Monumentos arquitectónicos de la provincia de Granada", Miscelaneas. Historia-Arte-Arqueología. Primera Serie: La Antigüedad, C.S.I.C. e Instituto Diego Velázquez, Madrid. pp. 347-390.; ARRIBAS PALAU, A., MOLINA GONZÁLEZ, F. (1977), op.cit;; ARRIBAS PALAU, A. MOLINA, F. (1979a): El poblado de los Castillejos en las Peñas de los Gitanos (Montefrío, Granada): campaña de excavaciones de 1971: el corte n 1, Cuadernos de Prehistoria de la Universidad de Granada. Serie Monográfica 3, Granada.; ARRIBAS PALAU, A., MOLINA GONZÁLEZ, F. (1979b), "Nuevas aportaciones al inicio de la metalurgia en la Península Ibérica. El poblado de Los Castillejos de Montefrío (Granada)", en The origins of metallurgy in Atlantic Europe. Proceedings of the fifth Atlantic colloquium (Dublin $30^{\text {th }}$ March to $4^{\text {th }}$ April 1978), published by the Stationery Office (editor RYAN, Michael), Dublín. pp. 7-34. 
En último lugar dentro del conjunto de Las Peñas de los Gitanos también se encuentra el Cerro de El Castellón, donde podemos encontrar un poblado y una necrópolis medieval que ya son mencionados por Góngora en $1868^{8}$. El poblado tiene su inicio en el siglo VIII d.C., en una época de transición entre el mundo visigodo y la invasión por parte de los musulmanes, y mantiene su ocupación hasta al menos el siglo X d.C. La necrópolis se encuentra en la falda occidental del mencionado cerro, y cuenta con grandes cistas rectangulares formadas con lajas hincadas a los lados y cubiertas de losas planas.

\section{CONTEXTO ESTRATIGRÁFICO DEL MATERIAL}

Dentro de la amplia secuencia estratigráfica con la que cuenta el yacimiento de Los Castillejos, los niveles pertenecientes al Neolítico Antiguo Avanzado se localizan en la parte inferior de la misma, ya que son los primeros estratos de ocupación detectados para el sitio que se localizaron durante el transcurso de los últimos trabajos desarrollados entre 1991 y 1994 ?

No obstante la relación de campañas de excavación realizadas en el yacimiento es amplia, comenzando por la de Manuel de Góngora que fue el primero en dar a conocer públicamente la existencia en Las Peñas de los Gitanos de varios yacimientos atribuibles a diferentes cronologías ${ }^{10}$. Posteriormente en 1907, el investigador Manuel Gómez-Moreno afirmó haber localizado en la zona de Los Castillejos cerámica neolítica, siendo así el primero en detectar la existencia de este periodo en el yacimiento ${ }^{11}$. A continuación destaca Cayetano de Mergelina, quien excava la terraza donde se localiza el poblado encontrando cerámica neolítica en superficie, y distinguiendo una fase de ocupación donde aparecían materiales del mismo periodo $^{12}$.

8 GÓNGORA Y MARTÍNEZ, M. de. (1868), op.cit. pp. 86.

9 AFONSO MARRERO, J.A., MOLINA GONZÁLEZ, F., CÁMARA SERRANO, J.A., MORENO QUERO, M., RAMOS CORDERO, U., RODRÍGUEZ ARIZA, M 0 .O. (1996): "Espacio y tiempo. La secuencia en Los Castillejos de Las Peñas de Los Gitanos (Montefrío, Granada)", I Congrés del Neolitic a la Peninsula Ibérica. Formació e implantació de les comunitats agricoles (Gavà-Bellaterra, 1995). Actes. Vol. 1. (J. Bosch, M. Molist, Orgs.), Rubricatum 1:1, Gavà. pp. 297-304.; CÁMARA SERRANO, J. A., AFONSO MARRERO, J. A., MOLINA GONZÁLEZ, F. (e.p.): La ocupación de Las Peñas de los Gitanos (Montefrío, Granada) desde el neolítico al mundo romano. Asentamiento y ritual funerario.; CÁMARA, J. A., (et al). (2005), "La cronología absoluta de los Castillejos en la Peña de los Gitanos" en Actas III Congreso del Neolítico en la Peninsula Ibérica:( Santander, 5 a 8 de octubre de 2003). Editorial Universidad de Cantabria, Santander. pp. 297-304.; RAMOS, U., AFONSO, J.A., CÁMARA, J.A., MOLINA, F., MORENO, M. (1997): Trabajos de acondicionamiento y estudio científico en el yacimiento de Los Castillejos en Las Peñas de los Gitanos (Montefrío, Granada), Anuario Arqueológico de Andalucía 1993:III, Sevilla. pp. 246-252.

10 GÓNGORA Y MARTÍNEZ, M. de. (1868), op.cit. pp. 57-88.

11 GÓMEZ-MORENO, M. (1949), op.cit. pp. 355.

12 MERGELINA, C. (1945-1946), op.cit. pp. 16 y 20. 
A mediados de los años cuarenta será Miguel Tarradell quien excave en Los Castillejos hasta alcanzar la roca madre, localizando multitud de restos cerámicos que a su juicio debían datarse en el Bronce $\mathrm{I}^{13}$. Cronología esta última que fue anulada y modificada primero por Antonio Arribas y Fernando Molina ${ }^{14}$ y posteriormente por Auxilio Moreno Onorato en $1982^{15}$ tras conocer los resultados de las excavaciones realizadas en el yacimiento en 1971 y 1974 por parte del Departamento de Prehistoria y Arqueología de la Universidad de Granada. Dichos trabajos dieron pie a una refasificación por la cual los materiales recién obtenidos y los desenterrados por Tarradell pasaron a considerarse como pertenecientes al Neolítico Final y Tardío ${ }^{16}$. Finalmente entre 1991 y 1994 la Universidad de Granada continuó con los trabajos de excavación, lo que permitió alcanzar de esta manera las fases iniciales del neolítico, completando al mismo tiempo la secuencia general de dicho periodo ${ }^{17}$.

Esta última campaña profundizó en los estratos neolíticos ya conocidos alcanzando niveles que no habían sido detectados con anterioridad, y que se clasificaron como Neolítico Antiguo. Este hecho provocó que la periodización de la secuencia cultural del yacimiento tuviera que redefinirse y ampliarse hasta alcanzar un total de nueve periodos prehistóricos, con sus correspondientes fases, que abarcan desde el Neolítico Antiguo hasta el Bronce Antiguo ${ }^{18}$, y a los que habría que sumar otros dos periodos históricos, uno perteneciente al mundo íbero-romano y otro a la Edad Media ${ }^{19}$. Este yacimiento abarca por lo tanto un amplio periodo cronocultural aunque no obstante el estudio que se está realizando en la actualidad se centra en las cerámicas decoradas de la fase 1 a la 4 del periodo I, es decir, en las cerámicas del Neolítico Antiguo Avanzado que han sido datadas en los últimos siglos del VI m. a.C. (5400 - 5150 cal. a.C.).

13 TARRADELL, M. (1952), op.cit. pp. 49-80.

14 ARRIBAS PALAU, A. MOLINA, F. (1979a), op.cit.

15 MORENO, Mª. A. (1982), op.cit. pp. 235-266.

16 MORENO, Ma. A. (1982), op.cit. pp. 238-239; ARRIBAS PALAU, A., MOLINA GONZÁLEZ, F. (1977), op.cit. pp. 391-399; ARRIBAS PALAU, A. MOLINA, F. (1979a), op.cit.

17 CÁMARA SERRANO, J. A., AFONSO MARRERO, J. A., MOLINA GONZÁLEZ, F. (e.p.), op.cit;; AFONSO MARRERO, J.A., MOLINA GONZÁLEZ, F., CÁMARA SERRANO, J.A., MORENO QUERO, M., RAMOS CORDERO, U., RODRÍGUEZ ARIZA, M 0 .O. (1996), op.cit.; pp. 297-298.; RAMOS, U., AFONSO, J.A., CÁMARA, J.A., MOLINA, F., MORENO, M. (1997): op.cit. pp. 265-271.; CÁMARA, J. A., (et al.) (2005), op.cit. pp. 297-304.

18 CÁMARA SERRANO, J. A., AFONSO MARRERO, J. A., MOLINA GONZÁLEZ, F. (e.p.), op.cit. pp. 12.

19 SÁNCHEZ ROMERO, M. (2000). Espacios de producción y uso de los útiles de piedra tallada del Neolitico. El poblado de Los Castillejos de Las Peñas de los Gitanos (Granada, España). Oxford: British Archaeological Reports. International Series 874. pp. 85. 
Por último en este apartado merece la pena mencionar, que la zona excavada para estas fases se ha interpretado como un área comunal donde se realizaban actividades de combustión, debido a la presencia de hogares, hogares-horno y anillos de barro y piedra. Además en ella se piensa que se realizarían actividades especializadas tales como la consumición de restos animales, el torrefactado del cereal o la talla a presión de los núcleos de sílex tras su calentamiento previo ${ }^{20}$.

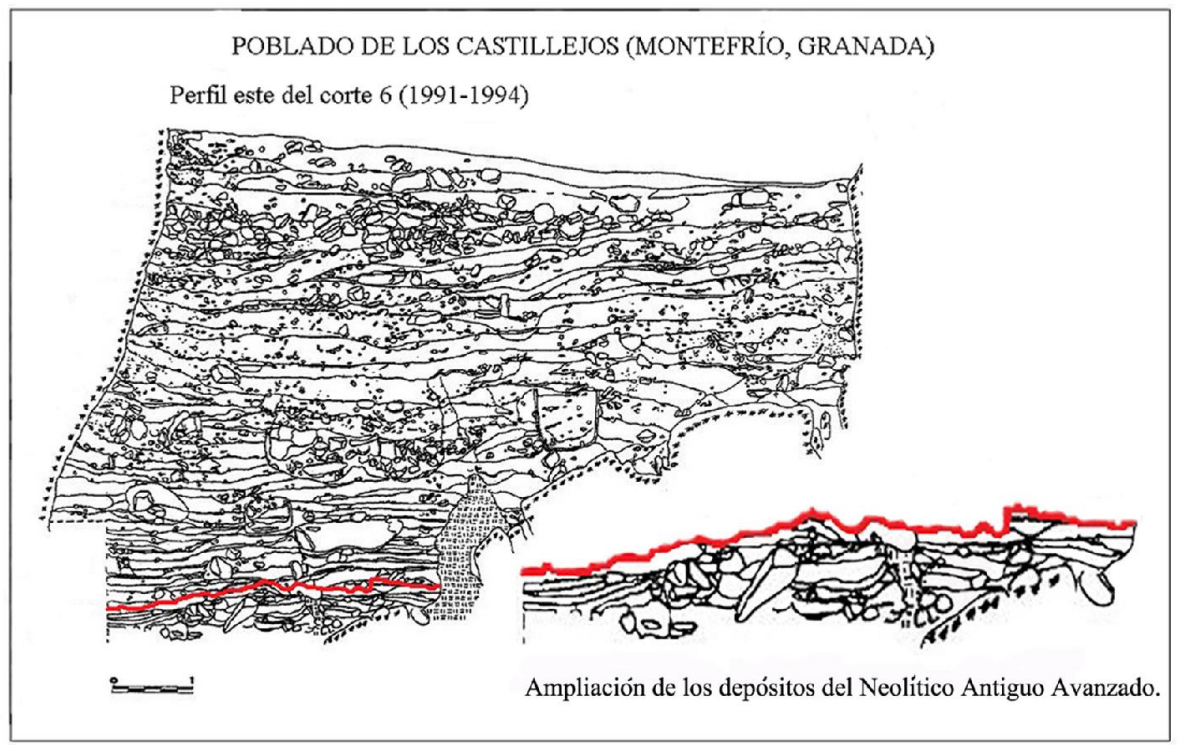

Fig. II Perfil estratigráfico del corte 6 con ampliación de los estratos estudiados.

\section{Metodología y RESUltados PRELIMINARES}

La metodología seguida para el estudio de las piezas cerámicas comenzó por la recopilación y lectura de la bibliografía relacionada con el yacimiento principalmente obras de trabajos arqueológicos anteriores, cuyas principales aportaciones ya han sido mencionadas en el apartado anterior.

En segundo lugar y después de conocer la historiografía del yacimiento se procedió a la localización de la totalidad de las piezas cerámicas, tanto de las decoradas como de las no decoradas, para proceder a su cuantificación y pesado y obtener de esta forma, la información necesaria para posteriormente poder realizar porcentajes y gráficos comparativos. Una vez realizado este

20 CÁMARA SERRANO, J. A., AFONSO MARRERO, J. A., MOLINA GONZÁLEZ, F. (e.p.), op.cit. pp. 13-17.; AFONSO MARRERO, J.A., MOLINA GONZÁLEZ, F., CÁMARA SERRANO, J.A., MORENO QUERO, M., RAMOS CORDERO, U., RODRÍGUEZ ARIZA, M 0 .O. (1996), op.cit.; pp. 297-298; RAMOS, U., AFONSO, J.A., CÁMARA, J.A., MOLINA, F., MORENO, M. (1997): op.cit. pp. 268. 
paso las piezas no decoradas no se volvieron a analizar, y la atención se centró en los fragmentos decorados ya que son éstos en los que se basa el estudio. De esta forma el siguiente paso consistió en la reproducción de éstos últimos, para ello por un lado se utilizó un escáner de funciones básicas marca HP donde las piezas fueron escaneadas a escala 1:1, y por otro lado se contó con el trabajo de Inés Casas López, dibujante profesional que se encargó de dibujar los perfiles de las piezas y de montar las imágenes, de tal forma que la pieza escaneada y el perfil aparecieran juntos en un mismo archivo.

Después de este proceso el análisis de las cerámicas de Los Castillejos continuó con el estudio de las decoraciones de las mismas, pretendiendo de esta manera comprobar su evolución en el Neolítico Antiguo Avanzado para el caso del ya mencionado yacimiento. De esta manera se comprobó que las técnicas decorativas básicas utilizadas eran: boquique, cordón, impregnación (incluyendo aguadas, almagras y pintadas), impresión, incisión, incrustación (de pasta blanca y en algunas ocasiones roja), y el modelado. Sin embargo, estas técnicas básicas frecuentemente aparecen combinadas unas con otras hasta alcanzar casi una treintena de diferentes tipos ornamentales.

Pero para observar la evolución de las decoraciones las cerámicas se estudiaron por grupos establecidos según la fase cronoestratigráfica a la que pertenecían y así se pudo llegar a una serie de conclusiones. Comenzando por el boquique se puede decir de él que se detecta desde el inicio de la secuencia, pero que pese a ello no se utilizó con demasiada frecuencia, a pesar de aparece asociado a la impregnación, la impresión, el cordón y la incrustación. Destaca sobre todo en la Fase 2 donde aparece en un total de tres fragmentos.

No sucede lo mismo con los cordones ya que aunque también se conocen desde la Fase 1 son mucho más numerosos, lo que indica que eran una técnica decorativa usada con gran frecuencia bien en forma de cordones lisos o asociados a otras técnicas, fundamentalmente la impresión. Los cordones aparecen en todas las fases pero sobre todo destacan en la fase 4 donde su número aumenta en gran medida respecto a las fases precedentes.

La impregnación es la otra gran técnica decorativa utilizada, y al igual que en el caso de los cordones aparece en todas las fases. Aunque se puede asociar a otras técnicas destaca cuando se encuentra en solitario, sobre todo en la Fase 1 que cuenta con nueve fragmentos, cifra que no será superada hasta llegar a la Fase 4 donde parece retomarse esta decoración con gran énfasis tras un periodo intermedio en el que había sido menos utilizada.

Las impresiones son otro grupo destacado aunque no tanto como los dos precedentes. Puede asociarse a otras técnicas, pero cuando aparece en solitario decorando las cerámicas destaca en la Fase 4. No obstante no sucede lo mismo si hablamos de las impresiones a peine o a cardium que destacan sobre todo en las Fases 1 y 2 , y que aunque continúan a lo largo de la secuencia lo hacen de forma menos numerosa y esporádica. 
Las incisiones aparecen desde la primera fase, pero aunque se asocien a otras decoraciones no llegan a destacar como una técnica decorativa importante en ninguna fase debido a los escasos fragmentos en los que se representa.

A continuación se realizó un análisis visual de las cerámicas decoradas donde se prestó especial atención a sus características tipológicas y tecnológicas. Así dentro de las primeras se tuvo en cuenta su forma y decoración, mientras que dentro de las características tecnológicas se ha valorado el color y el tratamiento de las superficies, y el estudio de las pastas, dentro de las cuales se ha estudiado su color, su compacidad y sus desgrasantes. Todo este análisis de características tuvo como resultado la creación de distintos grupos tecnológicos que muestran distintas formas de tratar las arcillas, y en general distintas formas de elaboración cerámica.

Este primer análisis macroscópico esta siendo en estos momentos contrastado mediante la observación de los mismos fragmentos a través de una lupa binocular. De esta forma y aunque aún no es definitivo se han establecido un total de cuatro grupos tecnológicos, alguno de ellos divididos en subgrupos, de los que a continuación se hace una breve referencia:

- Grupo 1: Este grupo se caracteriza de forma general por tener unas matrices arcillosas y compactas que denotan el cuidado con el que se elaboraron durante el proceso de amasado. Este primer grupo ha sido dividido a su vez en dos subgrupos.

o Grupo 1A: Este subgrupo se caracteriza por la presencia de desgrasantes muy finos, inferiores a $1 \mathrm{~mm}$. de longitud, y por poseer una matriz muy compacta.

o Grupo 1B: Este subgrupo se asemeja al anterior ya que el tamaño de los desgrasantes sigue siendo muy fino, pero la clave para diferenciarlo ha sido la matriz, que deja de ser tan compacta como en el caso anterior debido al aumento de desgrasantes en ella.

- Grupo 2: Las cerámicas de este grupo se caracterizan por incluir en sus pastas desgrasantes principalmente de tamaño medio, es decir entre 1 y $2 \mathrm{~mm}$., aunque también presentan algunos finos dándole a la matriz un aspecto más magro que en los casos precedentes.

- Grupo 3: Las piezas de este grupo constan de desgrasantes de tipo medio y fino que dan un aspecto mucho más granulado a la pasta que en el caso precedente. Se trata de las cerámicas más magras de todo el conjunto estudiado.

- Grupo 4: En este último grupo tan solo se ha incluido hasta el momento una pieza cerámica que cuenta con abundantes desgrasantes finos y medios. No obstante los desgrasantes que aparecen en ella no son los comunes para el resto de fragmentos por lo que se trata de una pieza de especial interés que debe ser estudiada en profundidad para comprobar su procedencia ${ }^{21}$.

21 La gran mayoría de las piezas del yacimiento presentan una matriz muy homogénea compuesta 

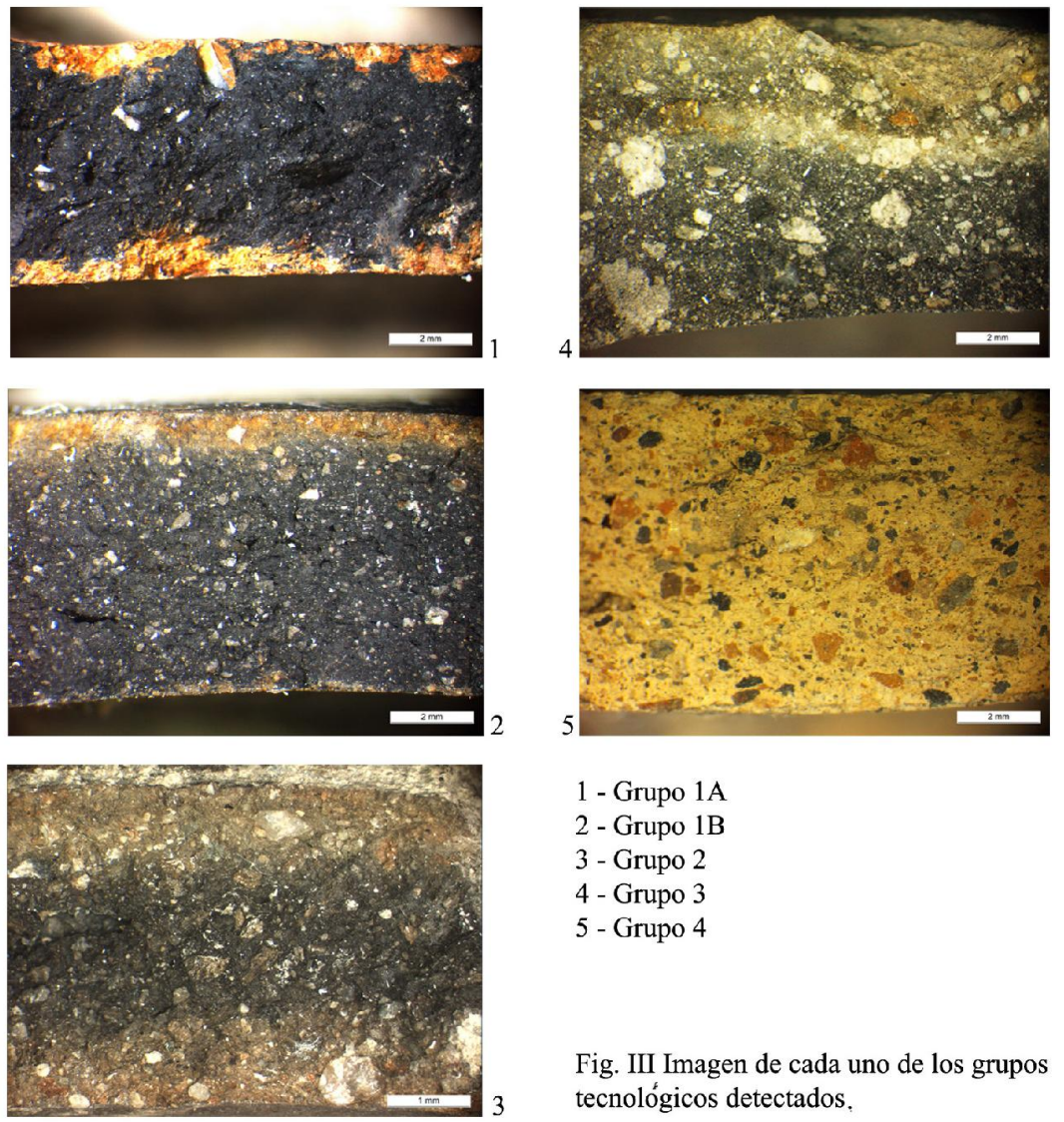

1 - Grupo $1 \mathrm{~A}$

2 - Grupo 1B

3 - Grupo 2

4 - Grupo 3

5 - Grupo 4

Fig. III Imagen de cada uno de los grupos tecnológicos detectados.

La metodología expuesta es la que se ha realizado hasta la fecha de escribir el presente artículo. No obstante el estudio de las cerámicas de Los Castillejos aún no está terminado por lo que la metodología también debe completarse, fundamentalmente con los resultados de difracción de rayos X y lámina delgada que en estos momentos se encuentran en proceso de elaboración. Pero aunque los resultados no estén disponibles actualmente, dentro de este apartado referido a la metodología si que se puede señalar que se seleccionaron aquellos fragmentos decorados que superaban los $3 \mathrm{~cm}$., para obtener muestras que posteriormente se destinarían a difracción de rayos $\mathrm{X}$ y a

por la arcilla y sus filosilicatos, cuarzos, feldespatos, materiales de arrastre, etc. sin embargo en este caso entre los desgrasantes destaca lo que parece identificarse como esquistos rojos. 
lámina delgada. Con el objetivo de obtener estas muestras se contó con la ayuda de unas tenazas comunes, con las que se cortaron dos muestras de cada cerámica, las de lámina delgada aproximadamente de $1 \mathrm{~cm}^{2}$ no sufrieron posteriores tratamientos, mientras que las muestras destinadas a difracción de rayos de $\mathrm{X}$ fueron molidas en un mortero de ágata hasta alcanzar un tamaño de unas 2 micras.

Finalmente se realizaron los difractogramas de las muestras anteriormente mencionadas ${ }^{22}$. Para ello en primer lugar se vertía y compactaba el contenido de las mismas en un portamuestras, a continuación éste se acoplaba al difractómetro gracias a un imán, y posteriormente desde un equipo informático se ponía en funcionamiento seleccionando el tipo de barrido. Una vez obtenido el difractograma se procedía a repetir la operación con otra muestra, y mientras se analizaba se podía ir comprobando los picos del anterior resultado con una base de datos de minerales del centro, que permitía ir identificando los reflejos cristalinos de los diferentes minerales detectados.

La metodología explicada en el presente artículo no supone más que el punto de partida de un análisis cerámico de mayores proporciones, por lo que se debe entender que las conclusiones que se pueden sacar son limitadas y abiertas, ya que tal y como se ha comentado anteriormente el estudio de las cerámicas aún no está concluido. No obstante, se debe valorar cada uno de los pasos dados y explicados a lo largo del artículo, puesto que cada uno de ellos ha proporcionado información y ha permitido que el siguiente pudiera llevarse a cabo. Así pues la historiografía ha sido el punto de partida básico que permitió conocer el yacimiento y todos los trabajos que en él se han desarrollado, la localización, cuantificación y pesado de los materiales facilitó el primer contacto con las cerámicas, lo que viene a favorecer cada uno de los pasos dados posteriormente.

La reproducción de las cerámicas también ayudó a visualizar las decoraciones a la hora de comprobar su evolución, y a su vez la información obtenida de las decoraciones debe de asociarse a las características tipológicas y tecnológicas de las pastas, ya que de esta forma se podrán comprender mejor los procesos de manufacturación y la utilidad de las diferentes vasijas. Debe de incorporarse también a esta metodología los futuros resultados de difracción de rayos X y lámina delgada, que proporcionarán información acerca de los componentes de la pasta cerámica y que pueden ayudar a definir las áreas de captación de la arcilla bien si ésta es de origen local al reflejar la geología del entorno, o si es de origen foráneo al yacimiento si la pasta cerámica procede de otras arcillas que no se localizan en las inmediaciones.

22 Los difractogramas se realizaron en el Centro de Instrumentación Científica de Granada, concretamente en el Laboratorio de Difracción de Rayos X y con la ayuda del personal que allí trabaja. 


\section{REFERENCIAS BIBLIOGRÁFICAS}

AFONSO MARRERO, J.A., MOLINA GONZÁLEZ, F., CÁMARA SERRANO, J.A., MORENO QUERO, M., RAMOS CORDERO, U., RODRÍGUEZ ARIZA, M 0 .O. (1996), Espacio y tiempo. La secuencia en Los Castillejos de Las Peñas de Los Gitanos (Montefrío, Granada), I Congrés del Neolitic a la Península Ibérica. Formació e implantació de les comunitats agrícoles (Gavà-Bellaterra, 1995). Actes. Vol. 1. (J. Bosch, M. Molist, Orgs.), Rubricatum 1:1, Gavà. pp. 297-304.

ARRIBAS PALAU, A., MOLINA GONZÁLEZ, F. (1977), "El poblado de Los Castillejos en Las Peñas de los Gitanos (Montefrío, Granada). Campañas de excavaciones de 1971 y 1974", XIV Congreso Nacional de Arqueología (Vitoria, 1975), Zaragoza. pp. 389-406.

ARRIBAS PALAU, A. MOLINA, F. (1979a), El poblado de los Castillejos en las Peñas de los Gitanos (Montefrio, Granada): campaña de excavaciones de 1971: el corte $n^{0} 1$, Cuadernos de Prehistoria de la Universidad de Granada. Serie Monográfica 3, Granada.

ARRIBAS PALAU, A., MOLINA GONZÁLEZ, F. (1979b), "Nuevas aportaciones al inicio de la metalurgia en la Península Ibérica. El poblado de Los Castillejos de Montefrío (Granada)", en The origins of metallurgy in Atlantic Europe. Proceedings of the fifth Atlantic colloquium (Dublin $30^{\text {th }}$ March to $4^{\text {th }}$ April 1978), published by the Stationery Office (editor RYAN, Michael), Dublín. pp. 7-34.

CÁMARA SERRANO, J. A., AFONSO MARRERO, J. A., MOLINA GONZÁLEZ, F. (e.p.): La ocupación de Las Peñas de los Gitanos Montefrío, Granada) desde el neolítico al mundo romano. Asentamiento y ritual funerario.

CÁMARA, J. A., MOLINA GONZÁLEZ, F., AFONSO MARRERO, J. A. (2005), "La cronología absoluta de los Castillejos en la Peña de los Gitanos" en Actas III Congreso del Neolitico en la Península Ibérica: (Santander, 5 a 8 de octubre de 2003). Editorial Universidad de Cantabria, Santander. pp. 297-304.

FERNÁNDEZ MARTÍNEZ, V. M. (1989), Teoría y método de la arqueología, Síntesis, Madrid.

GÓMEZ-MORENO, M. (1949), "Monumentos arquitectónicos de la provincia de Granada", Miscelaneas. Historia-Arte-Arqueología. Primera Serie: La Antigüedad, C.S.I.C. e Instituto Diego Velázquez, Madrid. pp. 347-390.

GÓNGORA Y MARTÍNEZ, M. de. (1868), Antigüedades prebistóricas de Andalucia, monumentos, inscripciones, armas, utensilios y otros importantes objetos pertenecientes a los tiempos más remotos de su población, Imprenta a cargo de C. Moro, Madrid.

MERGELINA, C. (1941-1942), "La estación arqueológica de Montefrío (Granada) I. Los dólmenes", Boletín del Seminario de Estudios de 
Arte y Arqueología VIII (B. S. A. A.), Valladolid. pp. 33-106.

MERGELINA，C. (1945-1946), "La estación arqueológica de Montefrío (Granada) II. La acrópoli de Guirrete (Los Castillejos)", Boletín del Seminario de Estudios de Arte y Arqueología XII, Valladolid. pp. 15-26.

MORENO, $\mathrm{M}^{\mathrm{a}}$. A. (1982), "Los materiales arqueológicos del poblado de Los Castillejos y Cueva Alta (Montefrío) procedente de las excavaciones de 1946 y 1947", Cuadernos de Prehistoria de la Universidad de Granada 7, Granada. pp. 235-266.

ORTON, C., TYERS, P., VINCE, A. (1997), La cerámica en Arqueología. Crítica. Barcelona.

RAMOS, U., AFONSO, J.A., CÁMARA, J.A., MOLINA, F., MORENO, M. (1997): Trabajos de acondicionamiento y estudio científico en el yacimiento de Los Castillejos en Las Peñas de los Gitanos (Montefrío, Granada), Anuario Arqueológico de Andalucia 1993:III, Sevilla. pp. 246-252.

SÁNCHEZ ROMERO, M. (2000), Espacios de producción y uso de los útiles de piedra tallada del Neolitico. El poblado de Los Castillejos de Las Peñas de los Gitanos (Granada, España). Oxford: British Archaeological Reports. International Series 874.

TARRADELL, M. (1952), "La Edad del Bronce en Montefrío (Granada). Resultados de las excavaciones en yacimientos de las Peñas de los Gitanos", Ampurias XIV, Barcelona. pp. 49-80.

TORRE SANTANA, Ma del P. (1984), "La Cueva de las Tontas en la estación arqueológica de Las Peñas de los Gitanos (Montefrío, Granada)", Cuadernos de Prehistoria de la Universidad de Granada 9, Granada. pp. 85-96. 
